\title{
AVALIAÇÃO DE JOGOS ELETRÔNICOS PARA USO NA PRÁTICA PEDAGÓGICA: ULTRAPASSANDO A ESCOLHA BASEADA NO BOM SENSO
}

\author{
Lúcia Regina Goulart Vilarinho, Fundação Cesgranrio, luciagvilarinho@gmail.com \\ Mariana Pinho Leite, Fundação Cesgranrio, marianapinho@gmail.com
}

\begin{abstract}
Resumo. O uso de jogos eletrônicos em sala de aula não é uma prática usual e quando ocorre nem sempre é precedido de uma reflexão crítica consistente, baseada em processos avaliativos sustentados por instrumentos de avaliação de jogos, adequadamente validados. Considerando esta problemática, desenvolveu-se um estudo cujo objetivo foi construir e validar um instrumento de avaliação de jogos eletrônicos educacionais ou de entretenimento - para uso em atividades pedagógicas. A partir da revisão da literatura sobre instrumentos de avaliação de jogos, foi elaborado e validado um instrumento, tendo-se como metodologia a abordagem centrada em especialistas. Como resultado principal do estudo destaca-se a relevância do instrumento para apoiar educadores na seleção de jogos a serem utilizados em situações de ensino-aprendizagem.
\end{abstract}

Palavras-chave: Educação. Jogos eletrônicos. Avaliação.

\begin{abstract}
The use of electronic games in the classroom is not an usual practice and when it occurs it is not always preceded by a critical reflection consistent, based on evaluative processes supported by evaluation tools of games, appropriately validated. Considering this problem, was developed a study which objective was to build and validate a tool for evaluation of electronic educational games or entertainment to be used in educational activities. The instrument was developed based on a review of the literature on evaluation tools of games and a methodology of experts-centered approach. As a main result of the study can be highlighted the relevance of the instrument built to support educators in selecting games to be used in teaching-learning situations.
\end{abstract}

Key-words: Education. Eletronic games. Evaluation.

\section{Introdução}

A busca de práticas pedagógicas mais dinâmicas e interativas, que privilegiem a pesquisa e diferentes formas de colaboração, tem sido objeto de interesse de educadores e instituições de ensino em todos os níveis. No âmbito deste desafio emergem evidências de que os jogos estimulam o interesse do estudante e a sua participação nas tarefas escolares (SAVI; 2010).

Salen e Zimmerman (2003, p. 96) registram que o jogo é "um sistema em que os jogadores se engajam em um conflito artificial, definido por regras, cujo resultado é quantificável”. Já Abt (1970, apud SALEN; ZIMMERMAN, 2012, p. 90-91) conceitua o termo como "uma atividade entre dois ou mais tomadores de decisão independentes, buscando alcançar seus objetivos em um contexto limitador [...], um jogo é um contexto com regras entre os adversários tentando conquistar objetivos".

Visualizados em uma perspectiva que vai além do entretenimento, os jogos podem ser bons recursos para ensinar e avaliar, porque exigem resolução de problemas 
e pensamento crítico (SHAFFER, 2006), além de colaboração e negociação, habilidades atualmente consideradas indispensáveis, mas bastante ausentes de muitas salas de aula.

$\mathrm{Na}$ contemporaneidade, os docentes encontram uma grande variedade de jogos, desde os tradicionais - de mesa, de caneta e papel, de cartas, de tabuleiro - até os eletrônicos, estes exercendo grande sedução entre alunos de qualquer idade, especialmente aqueles que Prensky (2012) denominou de nativos digitais, ou seja, sujeitos que já nasceram sob a égide das tecnologias digitais de informação.

Apesar das possibilidades dos jogos eletrônicos em termos de desafiar e promover a interação dos jogadores a partir dos problemas apresentados, Hays (2005) admite que a seleção desses recursos para uso em situações de ensino-aprendizagem não tem sido feita com base em critérios de avaliação pré-estabelecidos, ou seja, apoiados por instrumentos específicos de avaliação. De um modo geral, educadores selecionam os jogos de forma intuitiva, valendo-se do seu bom senso, desconsiderando aspectos mais amplos, que envolvem as necessidades de aprendizagem, a acessibilidade e/ou as deficiências de seus alunos (BASSANI et al, 2010) na sua interação com as possibilidades criadas pelo jogo. Tal problemática sugere que a introdução dos jogos eletrônicos na sala de aula merece ser direcionada pelo uso de instrumentos que subsidiem os docentes na avaliação / seleção dos jogos mais adequados aos seus propósitos educacionais.

\section{O estudo: objetivo, questões avaliativas e sua relevância}

Buscando auxiliar os educadores que utilizam esses recursos (ou pensam em utilizar) a refletirem criticamente sobre a sua seleção, admitiu-se que seria oportuna a construção / validação de um instrumento de avaliação de jogos eletrônicos que incluísse tanto as dimensões específicas do jogo, como também, as pedagógicas. É importante ressaltar que o estudo não discute possibilidades e vantagens do uso de jogos na Educação, pois isto está amplamente apontado na literatura pedagógica; ele se concentrou na construção e validação de um instrumento técnico, baseado em indicadores que apresentam íntima relação com as questões que usualmente são levantadas por um docente no seu processo seletivo de jogos aplicáveis à situação escolar.

A proposta avaliativa foi orientada pela seguinte questão: em que medida o instrumento elaborado é adequado / útil para apoiar educadores a avaliar e selecionar jogos eletrônicos a serem usados em situações de ensino-aprendizagem.

A relevância desta validação encontra respaldo em Kirriemuir; Mcfarlane (2004) e Freitas; Oliver (2006) quando afirmam que, mesmo com todo o interesse acerca dos jogos, são poucas as tentativas no sentido de desenvolver modelos capazes de avaliá-los para uso em situações de ensino-aprendizagem. A análise de alguns instrumentos de avaliação de jogos educacionais revelou que, em sua maioria, priorizam a avaliação de características técnicas em detrimento das características educacionais. Por outro lado, quando incluem a dimensão pedagógica, deixam de lado pontos extremamente importantes e característicos dos jogos, como a experiência que o usuário vive durante o jogo, conhecida como user experience (UX). Tais limitações reforçaram $\mathrm{o}$ desenvolvimento da presente proposta.

\section{Jogos: do simples entretenimento à complexidade dos jogos eletrônicos}

Os jogos, sejam eles tradicionais ou eletrônicos, possuem características comuns, como a presença de jogadores, regras, objetivos, estratégias, interatividade e 
uso de materiais. Os eletrônicos se diferenciam dos outros tipos de jogos por acontecerem em um ambiente digital, com a presença de elementos virtuais, simulações e diversidade de recursos. Neles, o jogador pode jogar sozinho, em dupla ou em grupo e o computador tem o potencial de ser o jogador rival. Goto $(2005$, p. 48$)$ entende que que os jogos eletrônicos: "podem ser descritos como a interação entre o jogador e as imagens que aparecem numa tela, mediada por um processador e uma interface física".

Neste estudo, o termo 'jogo eletrônico' se refere a jogos executados em um suporte computacional, tendo regras e um ambiente de interação com o usuário, de modo que as ações provocam reações no andamento do jogo até que seu objetivo seja alcançado.

Prensky (2012) entende que a utilização de jogos eletrônicos na sala de aula pode promover a aproximação entre os nativos digitais (alunos que já nasceram na cultura digital) e os imigrantes digitais (os professores com suas resistências às tecnologias). Os jogos ajudam o aluno a passar de espectador para pesquisador e o professor a tornar-se um guia que apoia o aluno na descoberta de seus próprios caminhos de aprendizagem.

Entre os jogos eletrônicos utilizados na situação de ensino-aprendizagem destaca-se o serious game, que tem a capacidade de simular qualquer tipo de situação. Podem ser usados para treinar profissionais em suas atividades ou situações críticas de seu trabalho, para conscientizar crianças, jovens e adultos sobre um determinado tema ou para simular situações (ZYDA, 2005). Segundo Blackman (2005), os serious games são jogos que extrapolam o nível do entretenimento, oferecendo outros tipos de experiências, como aprendizado e treinamento. São criados do mesmo modo que os jogos de entretenimento, incluindo: levantamento de objetivos, personagens e fases. $\mathrm{O}$ levantamento de competências e habilidades a serem desenvolvidas é etapa importante na criação do jogo e irá estruturar todas as demais, voltadas para tornar o jogo lúdico, interativo e prazeroso.

\section{Procedimentos Metodológicos}

A presente proposta tomou a Abordagem Centrada em Especialistas como base teórico-metodológica. Nesta abordagem a avaliação "depende basicamente dos conhecimentos específicos de um profissional para julgar uma instituição, um programa, um produto ou uma atividade" (WORTHEN; SANDERS; FITZPATRICK, 2004; p. 179). A opção por este tipo de abordagem se deu em função da necessidade de conjugar julgamentos de qualidade, emitidos por profissionais multidisciplinares das áreas de Educação, Avaliação e Jogos. De acordo com Worthen, Sanders e Fitzpatrick (2004), uma equipe de especialistas, ao se complementar de forma mutual, tem maior probabilidade de realizar avaliações mais consistentes e completas.

O estudo partiu de uma revisão da literatura, envolvendo a análise de 10 instrumentos de avaliação de jogos digitais e dela sobressaíram seis, registrados nos seguintes textos: (a) ISO, 2013; (b) Gladcheff et al, 2011; (c) LORI (NESBIT, BELFER; LEACOCK, 2009); (d) Game Flow; e E-Game Flow (FU; SU; YU (2009); e (f) um questionário de avaliação de jogos educacionais. A leitura acurada desses instrumentos permitiu a identificação das características recorrentes, isto é, dos aspectos que mais sobressaíram na análise que se propunham a fazer: (a) desafio e feedback - 4 indicações; (b) concentração, imersão, interação social e usabilidade - 3 recorrências; e (c) clareza dos objetivos, conteúdo, controle, habilidade do jogador - 2 indicações.

$\mathrm{Da}$ análise inicial inferiu-se que os itens mais destacados eram os relativos à experiência do usuário, entre eles: concentração, controle, desafio, imersão e interação 
social. Verificou-se, também, que aspectos como funcionalidade, manutenibilidade e portabilidade, relacionados à interface do jogo, e os pedagógicos, como alinhamento aos objetivos de aprendizagem e aos aspectos psicopedagógicos, não foram valorizados na maior parte dos instrumentos. Tal lacuna pode indicar uma despreocupação com as questões pedagógicas e/ou dificuldade de criar indicadores para avaliar a qualidade desses aspectos.

Há aspectos que não foram destacados na maior parte dos instrumentos, no entanto, para o caso de jogos eletrônicos pedagógicos são fundamentais, a saber: alinhamento com os objetivos de aprendizagem; design da apresentação; e motivação. É importante destacar que os aspectos pedagógicos propostos nos instrumentos estudados foram considerados insuficientes. Assim, foram criados seis indicadores na categoria pedagógica, a saber: adequação da linguagem; ampliação dos recursos cognitivos; estímulo à resolução de problemas; perspectiva interdisciplinar; presença de recursos de avaliação; uso de situações contextualizadas. Por fim, foi definido um indicador na categoria interface: ausência de erros técnicos.

As etapas seguintes se concentraram na construção do instrumento em si, o que incluiu a criação de novas categorias e dos seus respectivos indicadores, e na definição dos padrões de avaliação. Em seguida, foi elaborado o questionário de avaliação do instrumento a ser utilizado pelos especialistas no processo de validação.

Participaram da validação 10 especialistas com formação multidisciplinar, sendo quatro da área de Avaliação, três da área de Jogos e três da área de Educação. Em relação à formação acadêmica, cinco eram doutores, três mestres e dois possuíam especialização (pós-graduação lato sensu). Seis validadores se declararam especialistas em mais de uma área de estudo. O questionário utilizado pelos especialistas foi composto de seis perguntas fechadas e abertas, que incluíam o registro dos aspectos favoráveis e desfavoráveis do instrumento. Nele também era dada a possibilidade de justificar as respostas e solicitada a atribuição de uma nota relativa à sua adequação. Os especialistas receberam três documentos: carta de apresentação com as informações gerais sobre a validação, o quadro de critérios que fundamentou a construção do instrumento, e o próprio instrumento.

\section{Resultados do estudo}

Avaliar um jogo eletrônico, com vistas a determinar o seu potencial para uso em situação de ensino-aprendizagem, não é um ato simples. A seriedade de uma avaliação dessa natureza passa pela utilização de um instrumento construído especificamente para este fim, respaldado em um quadro de critérios no qual estejam registrados: categorias de avaliação (aquilo que vai ser avaliado); indicadores (desdobramentos das categorias); e desempenhos esperados do objeto em análise, ou seja, critérios ou padrões que vão nortear a avaliação. Segundo Worthen, Sanders e Fitzpatrick (2004, p. 695), critérios são: "indicadores de mérito que definem as características de um programa ou de uma implementação bem-sucedida". Ainda que possam aparecer explícitos nas próprias perguntas da avaliação, os critérios a serem usados para julgar o valor do objeto focalizado devem ser definidos com clareza.

O quadro de critérios que se segue resultou da análise realizada sobre os instrumentos de avaliação de jogos eletrônicos encontrados na literatura. Ele apresenta três dimensões a serem avaliadas: pedagógica, experiência do usuário e interface.

\section{Quadro de Critérios}




\begin{tabular}{|c|c|c|}
\hline Categoria & Indicadores & Padrões \\
\hline \multirow{11}{*}{ Pedagógica } & Adequação da linguagem & \multirow{21}{*}{$\begin{array}{l}1 \text { - muito ruim } \\
2 \text { - ruim; } \\
3 \text { - bom; } \\
4 \text { - muito bom. }\end{array}$} \\
\hline & Alinhamento com os objetivos de aprendizagem & \\
\hline & Ampliação dos recursos cognitivos & \\
\hline & Clareza dos objetivos & \\
\hline & Correção do conteúdo & \\
\hline & Estímulo à resolução de problemas & \\
\hline & Fornecimento de feedback & \\
\hline & Perspectiva interdisciplinar & \\
\hline & Presença de recursos de avaliação & \\
\hline & Promoção do desenvolvimento do sujeito & \\
\hline & Uso de situações contextualizadas & \\
\hline \multirow{7}{*}{$\begin{array}{c}\text { Experiência do } \\
\text { usuário }\end{array}$} & Capacidade de desafiar & \\
\hline & Capacidade de motivar & \\
\hline & Capacidade de reter a concentração & \\
\hline & Desenvolvimento do jogador & \\
\hline & Interação social & \\
\hline & Promoção da imersão & \\
\hline & Sensação de controle & \\
\hline \multirow{3}{*}{ Interface } & Ausência de erros técnicos & \\
\hline & Clareza das informações visuais & \\
\hline & Facilidade de navegação & \\
\hline
\end{tabular}

A categoria pedagógica se justifica pela força das questões educacionais quando se trata da avaliação de um jogo a ser inserido na prática pedagógica. Tem por objetivo avaliar se o jogo utiliza estratégias que mobilizem processos de aprendizagem e promovam o desenvolvimento cognitivo e social do sujeito. A experiência do usuário refere-se às experiências provocadas no jogador e que tornam o jogo mais interessante. Já a dimensão interface tem sua importância justificada por ser o elemento que se situa entre o jogador e o jogo. Interface pode ser um painel ou um sistema de botões e luzes, que fica situado entre dois elementos, garantindo a comunicação entre eles. Entende-se que esta categoria deve estar atrelada a indicadores de qualidade, quando aplicada a situações de ensino-aprendizagem, como funcionamento correto, sem erros, com fácil navegação e informações visuais claras.

$\mathrm{O}$ instrumento incluiu 20 afirmativas a serem julgadas conforme o grau de concordância do avaliador em relação a cada uma delas, cabendo escolher um dos quatro padrões da escala: (a) muito ruim, (0 a 25\%); (b) ruim (de 26 a 50\%); (c) bom (de 51 a 75\%); e (d) muito bom, quando o avaliador entender que o jogo atendeu plenamente ao que está registrado na afirmativa (76 a 100\%). Ao verificar que o jogo não concretiza o que é apresentado na afirmativa, o avaliador deve assinalar a opção muito ruim (0 a $25 \%)$.

Foi definido, também, que cada intervalo de percentuais possuía um valor de pontuação: muito ruim igual a 1 ponto; ruim, equivalente a 2 pontos; bom, 3 pontos; e muito bom, 4 pontos. Ao final do instrumento, o avaliador deve somar o total de pontos obtidos em cada dimensão avaliada e no computo global, fazendo a classificação do jogo de acordo com o sistema de pontuação proposto. Tal sistema tem por objetivo apresentar a classificação do jogo: uma estrela (jogo péssimo), duas estrelas (jogo ruim), três estrelas (jogo razoável), quatro estrelas (jogo bom / ótimo). Os jogos recomendados para uso são os classificados com quatro ou cinco estrelas, bom e ótimo, respectivamente. Porém, quando o jogo não atinge as três estrelas (razoável), o professor pode complementá-lo com outra atividade pedagógica. A seguir é apresentado o instrumento construído. 


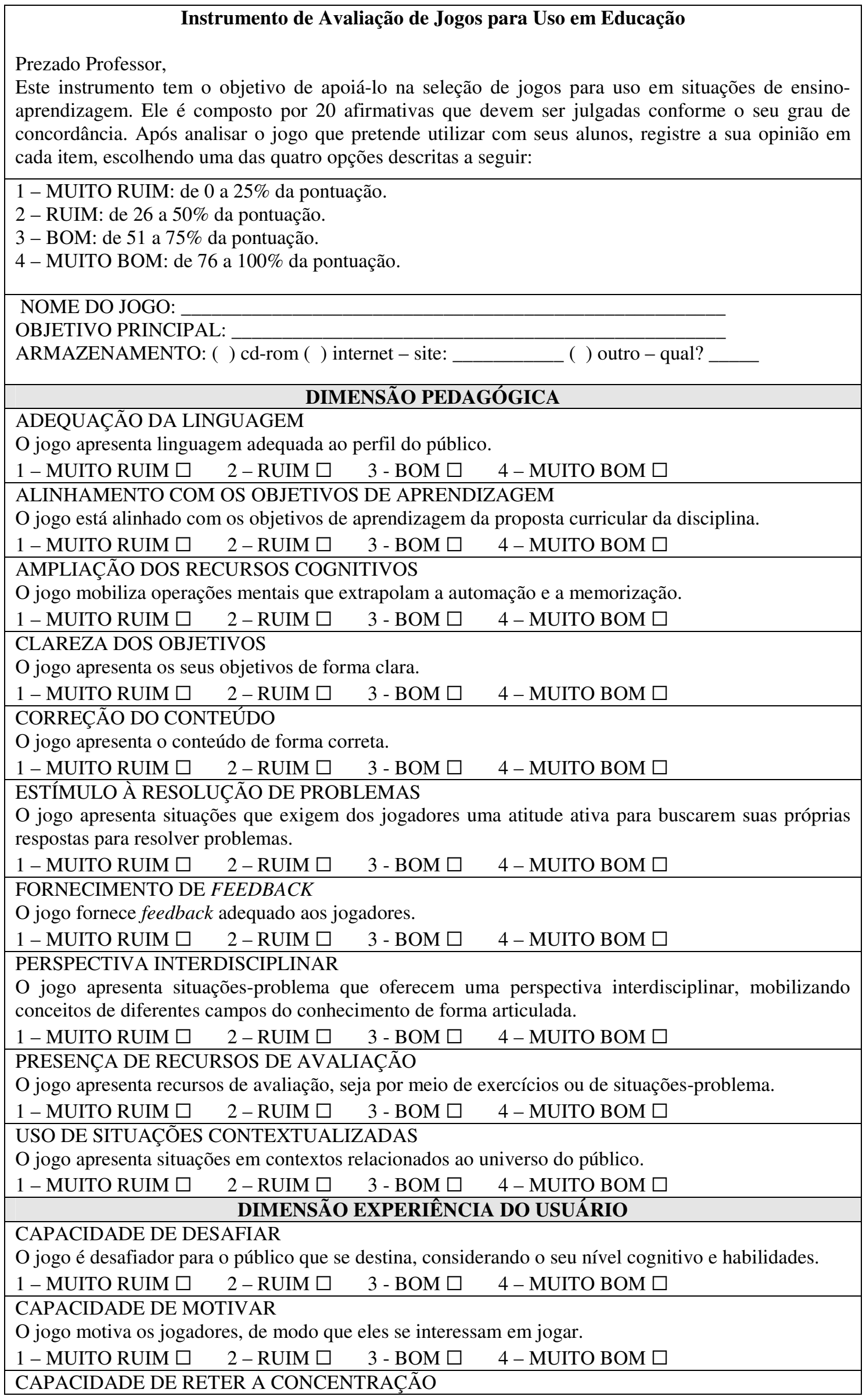




\begin{tabular}{|c|c|c|c|}
\hline \multicolumn{4}{|c|}{ O jogo exige e mantém a concentração dos jogadores. } \\
\hline \multirow{2}{*}{\multicolumn{4}{|c|}{$\begin{array}{l}\text { DESENVOLVIMENTO DO JOGADOR } \\
\text { O jogo apoia o desenvolvimento das habilidades dos jogadores, fazendo com que eles se sintam bem- } \\
\text { sucedidos com o seu progresso ao longo das fases/etapas. }\end{array}$}} \\
\hline & & & \\
\hline 1 - MUITO RUIM $\square$ & 2 - RUIM $\square$ & $3-$ BOM $\square$ & 4 - MUITO BOM $\square$ \\
\hline \multicolumn{4}{|c|}{ INTERAÇÃO SOCIAL } \\
\hline \multicolumn{4}{|c|}{ O jogo estimula a interação social, por meio da empatia, cooperação ou competição. } \\
\hline 1 - MUITO RUIM $\square$ & $2-$ RUIM $\square$ & $3-$ BOM $\square$ & 4 - MUITO BOM $\square$ \\
\hline \multirow{2}{*}{\multicolumn{4}{|c|}{$\begin{array}{l}\text { PROMOÇÃO DA IMERSÃO } \\
\text { O jogo envolve profundamente os jogadores diminuindo sua consciência em relação ao que está ao seu } \\
\text { redor e à sua noção de tempo. }\end{array}$}} \\
\hline & & & \\
\hline 1 - MUITO RUIM $\square$ & 2 - RUIM $\square$ & $3-$ BOM $\square$ & 4 - MUITO BOM $\square$ \\
\hline \multicolumn{4}{|c|}{ SENSAÇÃO DE CONTROLE } \\
\hline \multicolumn{4}{|c|}{ O jogo dá aos jogadores a sensação de controle sobre suas ações. } \\
\hline 1 - MUITO RUIM $\square$ & 2 - RUIM $\square$ & $3-$ BOM $\square$ & 4 - MUITO BOM $\square$ \\
\hline \multicolumn{4}{|c|}{ DIMENSÃO INTERFACE } \\
\hline \multicolumn{4}{|c|}{ AUSÊNCIA DE ERROS TÉCNICOS } \\
\hline 1 - MUITO RUIM $\square$ & 2 - RUIM $\square$ & 3 - BOM $\square$ & 4 - MUITO BOM $\square$ \\
\hline \multicolumn{4}{|c|}{$\begin{array}{l}\text { O jogo possui apresentação visual clara, com o uso de fontes, imagens, cores e quantidade adequada de } \\
\text { informações por tela. }\end{array}$} \\
\hline 1 - MUITO RUIM $\square$ & 2 - RUIM $\square$ & 3 - BOM $\square$ & 4 - MUITO BOM $\square$ \\
\hline \multicolumn{4}{|c|}{ FACILIDADE DE NAVEGAÇÃO } \\
\hline 1 - MUITO RUIM $\square$ & 2 - RUIM $\square$ & 3 - BOM $\square$ & 4 - MUITO BOM $\square$ \\
\hline \multicolumn{4}{|c|}{$\begin{array}{l}\text { Após a utilização do instrumento, some os pontos atribuídos em cada opção que você escolheu e faça a } \\
\text { classificação do jogo de acordo com o sistema de pontuação a seguir. }\end{array}$} \\
\hline \multicolumn{4}{|c|}{$\rightarrow \square \square 20$ a $30-\star \square \square$ (péssimo) } \\
\hline \multicolumn{4}{|c|}{$\rightarrow \square \square 31$ a $50-\star \star \square \square$ (ruim) } \\
\hline \multicolumn{4}{|c|}{$\rightarrow \square \square 51$ a $69-\star \star \star \square \square$ (razoável) } \\
\hline \multicolumn{4}{|c|}{$\rightarrow \square \square 70$ a $89-\star \star \star \star \square \square($ bom) } \\
\hline \multicolumn{4}{|c|}{$\rightarrow \square 90$ a $100-\star \star \star \star \star \star \square \square$ (ótimo) } \\
\hline \multicolumn{4}{|c|}{$\begin{array}{l}\text { *ATENÇÃO! Os jogos recomendados para uso são os classificados com quatro estrelas (bom) ou } \\
\text { cinco (ótimo). Porém, caso um jogo avaliado tenha sido classificado com três estrelas (razoável), o } \\
\text { professor pode utilizá-lo, complementando com outra atividade pedagógica para atender aos aspectos } \\
\text { não atendidos pelo jogo. }\end{array}$} \\
\hline
\end{tabular}

\section{Validação do instrumento}

As indagações contidas no questionário de avaliação do instrumento foram: (a) você concorda com as categorias propostas no instrumento? (b) você acha que os indicadores julgam o que estão se propondo a avaliar? (c) você concorda com os padrões propostos no instrumento? (d) em sua opinião, quais são os aspectos favoráveis e desfavoráveis do instrumento? (e) você acha que a utilização do instrumento pode apoiar educadores na seleção de jogos eletrônicos para uso em situações de ensinoaprendizagem? (f) o instrumento está adequado em relação ao objetivo para o qual foi criado, ou seja, apoiar educadores na seleção de jogos eletrônicos para uso em situações de ensino-aprendizagem? Em todas elas foram dadas duas opções: sim e não, sendo pedida a justificativa da escolha da opção não. Ao final da avaliação, o especialista tinha de atribuir uma pontuação / conceito ao instrumento, escolhida em uma escala de cinco pontos: 1- péssimo; 2 - fraco; 3 - regular; 4 - bom; 5 - excelente. 
O objetivo desta validação foi contribuir para o desenvolvimento e melhoria do instrumento, ou como afirmam Worthen, Sanders e Fitzpatrick (2004, p. 498): "reduzir e sintetizar informações - dar sentido a elas - e permitir inferências sobre populações".

Sobre as categorias propostas no instrumento, todos os 10 especialistas concordaram com elas, validando, então, as dimensões: pedagógica, experiência do usuário e interface. Quanto aos indicadores, todos os validadores também responderam que estavam adequados. Cinco especialistas fizeram sugestões sobre a redação de alguns indicadores, as quais foram consideradas. Outras sugestões não se aplicavam.

Em relação aos padrões propostos no instrumento, nove dos 10 especialistas concordaram com eles. Um especialista, no entanto, fez as seguintes sugestões: "Concordo com os padrões, mas julgo ser mais adequado utilizar uma escala de quatro pontos, retirando, por exemplo, o item péssimo, da escala". Tal sugestão foi atendida e o instrumento adaptado para quatro padrões, a saber: muito ruim, ruim, bom e muito bom.

Um especialista registrou o seguinte comentário: "Não tem a opção da pessoa não saber responder?" No entanto, se houvesse a opção: "não sei avaliar', o jogo não teria uma soma adequada de pontos e isto, no final, prejudicaria a sua classificação. Com base nas respostas dos especialistas, foi possível inferir que o instrumento possui os critérios adequados para a avaliação.

No que tange aos aspectos favoráveis do instrumento, os especialistas indicaram as dimensões (ou categorias), os indicadores, a formatação e o tamanho do instrumento. Salientaram, também, a segurança que o instrumento oferece ao professor que deixa de escolher os jogos baseando-se no 'bom senso'. Foram registrados os seguintes comentários em relação a este aspecto: "organiza muito bem as decisões que o professor terá que tomar ao realizar seu planejamento"; "caso algum aspecto esteja falho no jogo avaliado, o professor pode complementar com outra atividade para garantir a aprendizagem"; "há grande demanda para este tipo de instrumento como recurso para o trabalho docente"; "o instrumento pode favorecer o professor na escolha dos jogos, especialmente nas categorias pedagógica e experiência do usuário".

As categorias/dimensões também foram consideradas adequadas pelos especialistas. Registraram: "permite uma análise detalhada dos jogos sob os pontos de vista pedagógico, lúdico e da experiência do jogador"; "um dos pontos favoráveis são as categorias abordadas"; "a abrangência das dimensões é um ponto favorável"; "aborda as diferentes dimensões que impactam a aprendizagem". Tais comentários reforçam a concordância dos especialistas em relação às categorias propostas. Os indicadores também foram citados como aspectos favoráveis do instrumento: "ponto positivo: os indicadores"; "a escolha dos indicadores".

Elogios genéricos também foram apresentados, como: "achei muito interessante essa iniciativa de trazer um apoio à decisão de utilização de jogos no ambiente educacional. Trabalho com jogos há muitos anos e tenho percebido o quanto agrega de valor aos métodos pedagógicos tradicionais"; "Gostei muito do instrumento, está muito claro e as questões são oportunas, para um instrumento de aplicação genérica"; "razoavelmente imparcial e pouco subjetivo, torna mais fácil julgar os méritos de um jogo de forma objetiva". Por fim, ainda foram situados como pontos positivos: "a formatação e o tamanho do instrumento".

Como aspectos desfavoráveis, os especialistas citaram quatro, sendo o primeiro: "exige certa motivação e disposição do docente para analisar cada indicador". Sobre este ponto entende-se que não se trata de um aspecto desfavorável do objeto em avaliação; ele se inscreve na sólida formação docente que garante a consciência crítica sobre a relevância do planejamento didático das atividades de ensino, o que inclui analisar cuidadosamente o material a ser usado na sala de aula. $O$ segundo ponto 
desfavorável veio inserido no seguinte comentário: "talvez fique difícil para o professor avaliar, se não tiver experiência com jogos. Sei que é justamente para ajudá-lo a analisar e usar jogos em sua prática pedagógica. Entretanto, penso que há um passo anterior, que é a familiaridade com jogos". Aqui também se percebe uma relação com o preparo específico do professor para enfrentar os desafios da cultura digital. É provável que o professor totalmente inexperiente em jogos digitais não se aventure a utilizá-los na sala de aula. No entanto, pode contar com a ajuda de seus alunos, especialmente se estes forem nativos digitais. Nesta mediação (aluno / jogo - professor) há espaço para o uso do instrumento em questão, pois um dos objetivos implícitos na sua construção foi estimular o uso de jogos em situações educacionais. Os demais comentários desfavoráveis foram considerados inexpressivos.

No que tange à possibilidade de utilização do instrumento como apoio a educadores na seleção de jogos digitais, todos os 10 professores responderam "sim", o que sugeriu a utilidade do instrumento. A última pergunta do questionário de avaliação do instrumento versava sobre a sua adequação ao objetivo para o qual fora criado; isto é, visava saber se ele poderia ser utilizado por educadores que pretendem trabalhar com jogos eletrônicos na situação formal de ensino-aprendizagem. No somatório dos pontos verificou-se que oito especialistas consideraram o instrumento excelente e dois situaram-no como bom. Tais resultados indicam a adequação e validade do instrumento.

\section{Considerações finais}

A inserção de jogos no contexto escolar causou muita polêmica no final do século XIX, por entender-se que o processo de aprendizagem não poderia comportar a brincadeira, o lúdico. No século XX, com renomados educadores e pensadores como Édouard Claparède (1873-1940) e Jean Piaget (1896-1980), o jogo entra definitivamente na escola, vindo a favorecer a motivação dos alunos para aprender. Hoje, em pleno século XXI, sob a égide da aceitação / valorização do jogo no contexto escolar, o desafio parece estar na formação do professor para trabalhar com os jogos eletrônicos que, de um modo geral, são rapidamente dominados pelos alunos. Esta formação passa necessariamente pela capacidade de o docente saber escolher, de forma crítica, os jogos que pode usar em sua(s) disciplina(s) e com o perfil de seus alunos. No caso do docente, mais importante que saber jogar, é saber escolher o jogo a ser utilizado. Uma escolha pedagógica exige reflexão muldimensional e o presente estudo apontou as três dimensões básicas que devem ser consideradas neste processo seletivo, a saber: (a) pedagógica - leva o professor a refletir sobre o que o jogo pode propor em termos de ampliação do conhecimento do aluno; (b) experiência do usuário - permite pensar sobre a capacidade de o jogo motivar, desafiar, favorecer a interação e a concentração; e (c) interface - facilita definir se o jogo se apresenta de forma inteligível, ou seja, se é de fácil entendimento para ser jogado.

Com este estudo espera-se contribuir para a inserção crítica de jogos eletrônicos no contexto escolar, auxiliando particularmente os docentes que se sentem inseguros em suas escolhas.

\section{Referências}

BASSANI, Patricia B. Scherer et al. Usabilidade e acessibilidade no desenvolvimento de interfaces para a educação a distância. Renote - Revista Novas Tecnologias na Educação, v.8, n.1, EAD, 2010, p. 1-10. 
BLACKMAN, Sue. Serious Games... and Less!. ACM Digital Library, New York. v. 39, n. 1, 2005, p. $12-16$.

FREITAS, Sara de; OLIVER, Michaela. How can exploratory learning with games and simulations within the curriculum be most effectively evaluated? Comput. Educ. v. 46, n. 3, 2006, p. 249-264.

FU, Fong-Ling; SU, Rong-chang; YU, Sheng-Chin. EGameFlow: a scale to measure learners' enjoyment of e-learning games. Computers in Entertainment, Oxford, v. 52, n. 1, jan. 2009, p. 101-112.

GLADCHEFF, Ana Paula; ZUFFI, Edna Maura; SILVA, Menezes Dilma. Um instrumento para avaliação da qualidade de softwares educacionais de matemática para o ensino fundamental. In: CONGRESSO DA SOCIEDADE BRASILEIRA DE COMPUTAÇÃO E WORKSHOP DE INFORMÁTICA NA ESCOLA, 7, 2011, Fortaleza, Anais... Fortaleza, 2011.

GOTO, Marcel R. Evoluindo a diversão: EGM Brasil. São Paulo: 35 Editora, 2005.

HAYS, Robert T. The effectiveness of instructional games: a literature review and discussion. Orlando: Naval Air Warfare Center Training System Division, 2005.

ISO. INTERNATIONAL ORGANIZATION FOR STANDARDIZATION. ISO 9241. Suíça, 2013. Disponível em: <http://www.iso.org/iso/en>. Acesso em: 19 dez. 2013.

KIRRIEMUIR, John; MCFARLANE, Angela. Literature review in games and learning. Bristol: Futurelab, 2004.

NESBIT, John; BELFER, Karen; LEACOCK, Tracey. Learning object review instrument (LORI): user manual. [S. 1.], 2009. Disponível em:

$<$ http://www.transplantedgoose.net/gradstudies/educ892/LORI1.5.pdf>. Acesso em: 3 fev. 2014.

PRENSKY, Marc. Aprendizagem baseada em jogos digitais. São Paulo: Senac, SP, 2012.

SALEN, Katie; ZIMMERMAN, Eric. Rules of play: game design fundamentals. Massachusetts: The MIT Press, 2003.

SAVI, Rafael et al. Proposta de um modelo de avaliação de jogos educacionais.

RENOTE: Revista Novas Tecnologias na Educação, v. 8, n. 3, dez. 2010.

SHAFFER, David Williamson. How computers games help children learn. New York: Palgrave MacMillan, 2006.

WORTHEN, Blaine R.; SANDERS, James L.; FITZPATRICK, Jody R. Avaliação de programas: concepções e práticas. São Paulo: Ed. Gente, 2004. 
ZYDA, Michael. From visual simulation to virtual reality to games. Computer. [S. 1.], v. 38, n. 9, p. 25-32, 2005. 